\title{
To what extent can mutual shifting of folded carbonaceous walls in slit-like pores affect their adsorption properties?
}

Article

Accepted Version

Furmaniak, S., Terzyk, A. P., Gauden, P. A., Włoch, J., Kowalczyk, P., Werengowska-Ciećwierz, K., Wiśniewski, M. and Harris, P. J. F. (2015) To what extent can mutual shifting of folded carbonaceous walls in slit-like pores affect their adsorption properties? Journal of Physics: Condensed Matter, 28 (1). 015002. ISSN 1361-648X doi:

https://doi.org/10.1088/0953-8984/28/1/015002 Available at https://centaur.reading.ac.uk/47315/

It is advisable to refer to the publisher's version if you intend to cite from the work. See Guidance on citing.

To link to this article DOI: http://dx.doi.org/10.1088/0953-8984/28/1/015002

Publisher: Institute of Physics Publishing

All outputs in CentAUR are protected by Intellectual Property Rights law, including copyright law. Copyright and IPR is retained by the creators or other copyright holders. Terms and conditions for use of this material are defined in the End User Agreement. 


\section{CentAUR}

Central Archive at the University of Reading

Reading's research outputs online 


\section{To what extent can mutual shifting of folded carbonaceous walls in slit-like pores affect their adsorption properties?}

Sylwester Furmaniak ${ }^{1 *}$, Artur P. Terzyk ${ }^{1}$, Piotr A. Gauden ${ }^{1}$, Jerzy Włoch ${ }^{2}$, Piotr Kowalczyk ${ }^{3}$, Karolina Werengowska-Ciećwierz ${ }^{1}$, Marek Wiśniewski ${ }^{1,4}$ and Peter J.F. Harris ${ }^{5}$

[1] Faculty of Chemistry, Physicochemistry of Carbon Materials Research Group, Nicolaus Copernicus University in Toruń, Gagarin St. 7, 87-100 Toruń, Poland

[2] Faculty of Chemistry, Synthesis and Modification of Carbon Materials Research Group, Nicolaus Copernicus University in Toruń, Gagarin St. 7, 87-100 Toruń, Poland

[3] School of Engineering and Information Technology, Murdoch University, Murdoch, Western Australia 6150, Australia

[4] INVEST-TECH R\&D Center, 32-34 Plaska Street, 87-100 Toruń, Poland [5] Electron Microscopy Laboratory, University of Reading, Whiteknights, Reading RG6 6AF, UK

\footnotetext{
* corresponding author:

Sylwester Furmaniak

e-mail: sf@chem.umk.pl

tel. (+48) (56) 611-44-27

fax: (+48) (56) 654-24-77

url: http://www.chem.umk.pl/ aterzyk
} 


\begin{abstract}
We have performed systematic Monte Carlo studies on the influence of shifting the walls in slitlike systems constructed from folded graphene sheets on their adsorption properties. Specifically, we have analysed the effect on the mechanism of argon adsorption $(T=87 \mathrm{~K})$ and on adsorption and separation of three binary gas mixtures: $\mathrm{CO}_{2} / \mathrm{N}_{2}, \mathrm{CO}_{2} / \mathrm{CH}_{4}$ and $\mathrm{CH}_{4} / \mathrm{N}_{2}(T=298 \mathrm{~K})$. The effects of the changes in interlayer distance were also determined. We show that folding of the walls significantly improves the adsorption and separation properties in comparison to ideal slitlike systems. Moreover, we demonstrate that mutual shift of sheets (for small interlayer distances) causes the appearance of small pores between opposite bulges. This causes an increase in vapour adsorption at low pressures. Due to overlapping of interactions with opposite walls causing an increase in adsorption energy, the mutual shift of sheets is also connected with the rise in efficiency of mixtures separation. The effects connected with sheet orientation vanish as the interlayer distance increases.
\end{abstract}

\title{
Key-words
}

folded graphene, slit-like pores, adsorption, gas mixtures separation, Monte Carlo simulations

\section{Introduction}

Recently, Lu and Dai [1] discussing developments in the synthesis of carbon nanomaterials for $\mathrm{CO}_{2}$ capture pointed out that "researchers should pay more attention to the indepth understanding of the physics and chemistry of carbon, which is highly important for the design and synthesis of tailored porous carbons as next-generation $\mathrm{CO}_{2}$ capture materials and model carbons for fundamental investigations". It is well-known that carbon materials exist in different nanoforms [Suarez-Martinez et al. 2012; Tomanek 2014, [2]]. Since new carbon nanomaterials are continuously being synthesized [2] the study of $\mathrm{CO}_{2}$ capture/separation mechanism in pores of different types is very important. Moreover, inspired by the experimental findings, much theoretical effort has been made to predict structures of carbon materials. For example novel carbon allotropes were predicted by Yang et al. [Yang et al. 2013] by optional substituting carbon atoms in diamond with carbon tetrahedrons. Nicolaï et al. [Nicolaï et al. 2015] proposed a new type of nanopores and membranes built from gyroid surfaces decorated with 
single-layer carbon materials. Zhou and Zeng [Zhou and Zeng 2012] classified polymorphic lowenergy $\mathrm{sp}^{3}$-hybridized carbon structures into three groups on the basis of different ways of stacking two (or more) out of five types (A-E) of buckled graphene layers. On the other hand, some "strange" carbon models first theoretically proposed, were next synthesized, for example pillared graphene [Dimitrakakis et al. 2008; Wesolowski and Terzyk 2011; Barker et al. 2012; Du et al 2011] or fullerenes distributed between graphene layers [Barker et al. 2012; Terzyk et al. 2009 Gupta et al. 2004, Kouloumpis et al. 2015].

In our recent study [3] we reported for the first time a detailed procedure for creating a simulation model of energetically stable, folded graphene-like pores and simulation results of $\mathrm{CO}_{2} / \mathrm{CH}_{4}$ and $\mathrm{CO}_{2} / \mathrm{N}_{2}$ separation using these structures. It was shown that folding of graphene structures is a very promising method to improve the separation of $\mathrm{CO}_{2}$ from mixtures with $\mathrm{CH}_{4}$ and $\mathrm{N}_{2}$. It is known that finite graphene sheets do not possess ideal flat one layer geometry. There are the possibilities of the deformation of those sheets by external factors (e.g. thermal fluctuation, mechanical forces, capillary pressure, ultrasonication). From the analysis of various reports in the literature one can see that graphene is one of the strongest materials tested, but because of its two-dimensional nature, graphene is strong only under tension and soft under compression [Zheng et al. 2011]. Besides the physical properties of graphene in the pristine form, the behaviour of graphene under strain also attracts the attention of scientific community. Depending on how the graphene is disordered, the different forms of the topology have been observed, for example via microscopy measurements, i.e. curved graphene flasks [Liu et al. 2013; Li and Östling 2013], ripples [Bao et al. 2009; Fasolino et al. 2007; Paronyan et al. 2011], the buckling of graphene nanoribbons [Schniepp et al 2008], folded edges [Meyer et al. 2007; Li and Östling 2013; Liu et al. 2009; Rotkin and Gogotsi 2002; Roy et al. 1998; Wen et al. 2012; Kim et al. 2011; Wang et al. 2015; Yan et al. 2013] and folded and curved bilayer graphene structures [Harris 2012; Harris et al. 2014]. The existence of those deformations increases the effective surface and ehange changes, for example, their adsorption and electrochemical properties [Li and Östling 2013; Yu et al. 2015; Wang and Zhao 2015; Kim et al. 2011; Baimova et al. 2014]. Liu et al. [Liu et al. 2013; Li and Östling 2013] obtained a mesoporous curved graphene structures with a pore size ranging from 2 to $25 \mathrm{~nm}$. The ripples at the nanoscale can be produced in a controlled way by means of a simple thermal manipulation [Bao et al. 2009]. Others also confirmed the existence of those structures [Fasolino et al. 2007; Paronyan et al. 2011]. On the other hand, 
Schniepp et al. [Schniepp et al 2008] investigated the bending properties of single functionalized graphene sheets with the tip of an atomic force microscope. Individual sheets are transformed by those authors from a flat into a buckling configuration (with a nearly sinusoidal profile). Sheets can be reversibly folded and unfolded multiple times, and the folding always occurs at the same location. Folded edges are unique to graphene, including graphene sheets of a few atomic layers, and 2D structures in general [Meyer et al. 2007; Li and Östling 2013]. The folded graphene edges have a nanotubelike structure, which possess unusual electronic properties [Liu et al. 2009] and mechanical stability resulting from the van der Waals attraction of folded graphene [Rotkin and Gogotsi 2002]. The surface layers of graphite can be folded by scratching using a scanning tip [Roy et al. 1998] or by high temperature annealing [Liu et al. 2009]. Wen et al. [Wen et al. 2012] developed a novel route to realize uniform doping in graphene which gives rise to highly crumpled graphene nanosheets. Kim et al. [Kim et al. 2011] presented experimental results that folded structures in graphene, termed grafold, exist, and their formations can be controlled by introducing anisotropic surface curvature during graphene synthesis or transfer processes. Wang et al. [Wang et al. 2015] created uniform arrays of graphene crumples on the centimetre scale by controlling simple thermal processing parameters without compromising the electrical properties of graphene. Highly crumpled graphene sheets have been prepared by Yan and co-workers [Yan et al. 2013] by a facile and rapid route through freezing a chemically reduced graphene oxide aqueous suspension with liquid nitrogen. Zhang et al. [Zhang et al. 2015] presented a simple and green but very efficient approach using two standard and simple industry steps to make threedimensional graphene-based porous materials at the bulk scale, with ultrahigh specific surface area $\left(3523 \mathrm{~m}^{2} / \mathrm{g}\right)$ and excellent bulk conductivity. These authors concluded that these materials consist of mainly defected/wrinkled single layer graphene sheets in the dimensional size-with the dimensions of a few nanometers, with at least some covalent bonds between each other. Moreover, it is well known that the application of so called two-phase polymerization [4] can leads to the creation of porous carbon containing channel like pores. In this case one can imagine that the pores are created from folded graphene walls, and now they are not parallel but shifted. One can therefore imagine that intermediate cases of porosity are also possible.

The problem of the deformation of the graphene sheets is also the subject of the intensive theoretical investigations [Zheng et al. 2011; Baimova et al. 2015; Chang et al. 2013; Cranford and Buehler 2011; Chen and Chen 2013; Chen et al. 2013; Baimova et al. 2012; Le and Woods 
2012; Le and Woods 2013; Ju et al. 2014; Neek-Amal and Peeters 2012; Ebrahimi 2015]. Zheng et al. [Zheng et al. 2011] studied the mechanical properties of grafold and an architecture of folded graphene nanoribbon via molecular dynamics simulations. In contrast to graphene, grafold is found to develop large deformations upon both tensile and compressive loading along the longitudinal direction. The mechanical properties of crumpled graphene via molecular dynamics (MD) simulation were studied by Baimova et al. [Baimova et al. 2015], Chang et al. [Chang et al. 2013], Cranford and Buehler [Cranford and Buehler 2011]. The problem of the formation corrugated graphene-like surfaces and their stability have been investigated using molecular dynamics simulation (peeling behaviour of a graphene sheet in adhesive contact with a corrugated copper substrate) [Chen and Chen 2013; Chen et al. 2013]. Strain-induced ripples in graphene nanoribbons with clamped edges are also investigated [Baimova et al. 2012]. Graphene nanoribbon folds with single and double closed edges are studied using density functional theory methods [Le and Woods 2012; Le and Woods 2013]. Ju et al. [Ju et al. 2014] theoretically considered thermal conductivity of folded graphene. Neek-Amal, Peeters, Ebrahimi investigated the efffect effect of grain boundary on the buckling of graphene nanoribbons via MD simulations [Neek-Amal and Peeters 2012; Ebrahimi 2015].

Corrugated systems were also studied as the nanocontainers [Tozzini and Pellegrini 2011; Dutta et al 2014]. Tozzini and Pellegrini [Tozzini and Pellegrini 2011] proposed a multilayer graphene-based device in which the storage and release of hydrogen were obtained by exploiting and controlling the corrugation of individual layers of graphene. Dutta et al [Dutta et al 2014] stated using van der Waals corrected density functional theory calculations that the differential between the adsorption of $\mathrm{CO}_{2}$ and $\mathrm{CH}_{4}$ is much higher on folded graphene sheets and at concave curvatures; this could possibly be exploited for $\mathrm{CH}_{4} / \mathrm{CO}_{2}$ flow separation and gas selective sensors. The discovery of folded graphene shows that the synthesis of carbon membranes containing folded graphene slit-like pores is plausible and it is interesting to investigate adsorption properties of this type of structures. Using density-functional theory calculations on a variety of model surfaces (folded and rippled graphene layers), Wood et al. [Wood et al. 2014] demonstrated that the low theoretical quantum capacitance of graphene-based electrodes can be significantly improved by altering local structural and morphological features.

The boxes constructed from the folded graphene sheets forming slit-like pores considered in our previous work [3] are also good model representation to study the effects of carbonaceous 
pore walls folding on adsorption mechanism. Dear professor Harris, could you add here a few sentences with suitable references about experimental proofs on folding of walls in real carbon?

Jagiello et al. [Jagiello and Olivier 2013a; Jagiello and Olivier 2013b; Jagiello et al. 2015] used the pores constructed from the corrugated walls during their NLDFT studies on pore size distribution (PSD) determination from the experimental adsorption isotherms for real carbons. They showed that due to the use of this approach the typical artefacts of the homogeneous slit pore model were eliminated. Also, the agreement of the new models with experimental data was significantly better than that of the standard slit model. Moreover, one of the available models in interactive SAIEUS software from [http://www.nldft.com/] for the analysis of adsorption (PSD calculation data using NLDFT theory) assumed corrugated geometry of pore walls.

In the present work, we present the results of systematic Monte Carlo studies on adsorption in model systems constructed from folded graphene sheets. The effects of degree of folding, mutual shifting of the walls and increase in the interlayer spacing are investigated. We consider Ar adsorption at its boiling point and adsorption and separation of model binary gas mixtures $\left(\mathrm{CO}_{2} / \mathrm{N}_{2}, \mathrm{CO}_{2} / \mathrm{CH}_{4}\right.$ and $\left.\mathrm{CH}_{4} / \mathrm{N}_{2}\right)$ at $298 \mathrm{~K}$. Finally we compare obtained results with those calculated previously for nanotubes and porous carbons.

\section{Calculation details}

\subsection{Simulation boxes}

We constructed multiplied slit-like systems using folded graphene sheets generated in our previous paper [3]. We used three thermodynamically equilibrated sheets with different amplitudes $(A)$ - see top of Figure 1. The following values of $A$ are considered: $0.50,0.75$ and $1.00 \mathrm{~nm}$ (we did not use the previously considered sheet with $A=0.25 \mathrm{~nm}$ since its adsorption properties are not significantly different form the flat graphene sheet [3]). In each simulation box with periodic boundary conditions in all three directions, four sheets were placed in a parallel arrangement. Next, every second sheet was shifted by $1 / 16,2 / 16,3 / 16$ or $4 / 16$ of the box length in the $x$ direction - see Figure 1. These relative shifts are denoted as $R S . R S=4 / 16$ is equivalent to antiparallel orientation of sheets. The initial effective distances between sheets $\left(H_{\text {eff }}\right)$ were selected so that the bulges of opposite carbonaceous structures were osculated for $R S=4 / 16$ (i.e. 
the channel-like systems). These interlayer distances were equal to $1.08 \mathrm{~nm}$ for $A=0.50 \mathrm{~nm}$, $1.52 \mathrm{~nm}$ for $A=0.75 \mathrm{~nm}$ and $1.91 \mathrm{~nm}$ for $A=1.00 \mathrm{~nm}$. The series of systems with the smallest initial values of $H_{e f f}$ are named H1. For each series the interlayer distance was next increased by $0.3 \mathrm{~nm}$ (obtained series are named H2) and by $0.6 \mathrm{~nm}$ (H3 series). Each system was labelled as A $x . x x \mathrm{H} y y \cdot y \mathrm{RS} z / z z$ where $x . x x$ is the amplitude, $y y \cdot y$ is the effective interlayer distance and $z / z z$ is the relative shift. In addition, for each series (for a given combination $\mathrm{n}$ of $A$ and $H_{\text {eff }}$ values) we also constructed the reference boxes using ideal flat graphene sheets $(A=0.00)$ placed at analogous distance. These systems are denoted as A0.00Hyy.y. The selected systems together with applied notation are presented in Figure 1. Table S1 in online supplementary data collects the sizes of all the considered simulation boxes.

The porosity of all the studied carbonaceous adsorbents was characterised by a geometrical method proposed by Bhattacharya and Gubbins (BG) [5]. The implementation of the method was described in detail elsewhere [6,7]. In order to calculate the histogram of pore diameters we applied the uniform grid of test points $(200 \times 200 \times 100)$ for each box.

\subsection{Simulations of argon adsorption isotherms}

Argon adsorption isotherms in all the considered systems were simulated using the hyper parallel tempering Monte Carlo method (HPTMC) proposed by Yan and de Pablo [8]. The simulations were performed for Ar boiling temperature $(T=87 \mathrm{~K})$. For each system, 93 replicas (corresponding to the relative pressures $\left(p / p_{s}\right.$, where $p$ and $p_{s}$ denote absolute and saturated vapour pressure at the given temperature, respectively) from $1.0 \times 10^{-10}$ to 1.0 ) were considered. The methodology of computations was described in detail previously [6]. The HPTMC simulations utilized $1 \times 10^{7}$ cycles (one cycle $=100$ attempts of the change of each replica state by (i) creation, (ii) annihilation or (iii) displacement of a randomly chosen atom with equal probabilities, and one attempt of a configuration swap between a pair of randomly chosen replicas). The first $2 \times 10^{6}$ cycles were discarded to guarantee equilibration. Table 1 collects the values of applied Lennard-Jones (LJ) parameters.

For each simulation point (value of relative pressure) the adsorption amount was calculated from the average number of $\mathrm{Ar}$ atoms in each replica $(\langle\operatorname{Ar}\rangle)$ : 
$a=\frac{\langle\mathrm{Ar}\rangle}{N_{C} \times M_{C}}$

where $N_{C}$ and $M_{C}$ are the number of carbon atoms in the box and the molar mass of carbon, respectively. In addition, for each isotherm the value of Henry's (or Langmuir) constant $\left(K_{H / L}\right)$ corresponding with low-pressure region was also determined. The details of these calculations are described in online supplementary data. Finally, the isosteric enthalpy of adsorption $\left(q^{s t}\right)$ was also calculated (from the theory of fluctuations) to study the energetics of the process.

\subsection{Simulations of gaseous mixtures adsorption}

We simulated the adsorption of three binary gas mixtures (important from practical point of view): $\mathrm{CO}_{2} / \mathrm{CH}_{4}, \mathrm{CO}_{2} / \mathrm{N}_{2}$ and $\mathrm{CH}_{4} / \mathrm{N}_{2}$. Their adsorption at $T=298 \mathrm{~K}$ in the above-described boxes was modelled using the grand canonical Monte Carlo method (GCMC) [14,15]. Simulations were performed for the total mixture pressure $p_{t o t}=0.1 \mathrm{MPa}$ (i.e. atmospheric pressure) and for the following mole fractions of components in the gaseous phase $(y): 0.0,0.01$, $0.025,0.05,0.1,0.2,0.3,0.4,0.5,0.6,0.7,0.8,0.9,0.95,0.975,0.99$ and 1.0. Additionally, in order to check the separation of the mixtures for other pressures, we simulated adsorption of equimolar mixtures $(y=0.5)$ at different total pressure values in the range $1.0 \times 10^{-6}-1.0 \mathrm{MPa}(30$ different values). The methodology of calculations was analogous to that described previously $[16,17]$. Each GCMC simulation run consisted of $2.5 \times 10^{8}$ iterations. The first $1.0 \times 10^{8}$ iterations were discarded to guarantee equilibration. One iteration was an attempt of the system state change by the randomly selected perturbation: (i) displacement and/or rotation of a randomly chosen molecule, (ii) creation of a new molecule, (iii) annihilation of a randomly chosen existing molecule or (iv) swap move with equal probabilities. We used equal probability for each perturbation to guarantee the condition of microscopic reversibility. The values of all the applied interaction parameters are collected in Table 1. Other computational details are given elsewhere $[11,16,18]$.

From the GCMC simulation results, we determined the average numbers of each kind of adsorbate molecules in the simulation box. These values were used for calculation of mole fractions of components in the adsorbed phase $\left(x_{i}\right)$. Finally, in order to illustrate the efficiency of mixture separation we also computed the values of equilibrium separation factors: 
$S_{1 / 2}=\frac{x_{1} / x_{2}}{y_{1} / y_{2}}$

The adsorbed phase is enriched in the $1^{\text {st }}$ component if $S_{1 / 2}>1$. Similarly as in the case of Ar adsorption, the isosteric enthalpy of adsorption was also calculated from the theory of fluctuations.

\section{Results and discussion}

\subsection{The structure of carbons and its influence on Ar adsorption}

Figure 2 collects the pore size histograms calculated using the BG method. Generally, one can observe three important effects. Shifting of the walls increase the pore size, simultaneously very small pores between opposite bulges of carbonaceous structures appear. The effect of the

appearance of small pores is especially visible on Figure 3 showing the visualization of pore sizes detected by the BG method. Obviously, an increase in interlayer distance causes the rise in pore diameters. Finally, one should notice that for some structures (especially for large $R S$ ) the pore diameter reaches the range of mesopores.

Figure 4 collects simulated Ar adsorption isotherms for carbons composed of folded graphene pores, and the isotherms for reference systems (i.e. ideal slit-like graphene pores). It is seen that with the rise in $A$ value maximum adsorption capacity decreases, compared to the reference systems. This is caused by the decrease in pore volumes during the folding of pore walls (see the size of simulation box in $x$ direction $\left(L_{b o x, x}\right)$ collected in Table S1 in online supplementary data). On the other hand, for all the studied cases in the low-pressure limits of adsorption isotherms we observe a rise in adsorption compared to the reference materials. We also observe a rise in pore filling pressure with the rise in $\mathrm{RS}$ value for a given series (i.e. for fixed $A$ and $H_{e f f}$ ) caused by the rise in pore diameters (see Figs. 2 and 3). Similar effect is observed if the pore diameter increases.

Considering the systems with the smallest interlayer distance between pore walls (i.e. H1 series) one can observe remarkable changes with the change in the RS value. Namely, with the rise in RS value the isotherms become steeper, and this effect is the most strongly visible for RS $=2 / 16$. The changes in the series $\mathrm{A} 0.50 \mathrm{H} 1.08$ and $\mathrm{A} 0.75 \mathrm{H} 1.52$ have similar qualitative character 
(Fig. 4). This can be explained by the analysis of the data collected on Figure 3, but it is especially visible on the starting frames of animations (see animation_01 and 02 in online supplementary data). One can notice for $\mathrm{RS}=2 / 16$ the appearance of narrow slit-like pores (remarkably increasing adsorption) vanishing for larger RS values. Additionally, in all the systems adsorption at low pressures also occurs at junctions and on high energy sites of the curved walls. A slightly different situation is observed for the series A1.00H1.91. Due to larger distance between walls, the effect of potential energy overlapping from opposite walls is not as strong as for smaller pores, and adsorption starts simultaneously on curved graphene sheets fragments (present in larger number) and at the junctions (see animation_03 in online supplementary data). Due to this effect, the influence of the RS change on adsorption isotherms is in this case small (Fig. 4).

Since the rise in pore width causes a simultaneous rise in the maximum adsorption capacity one can observe the change in adsorption mechanism (see animation_04, 05 and 06 in online supplementary data). In the case of the H2 series (Fig. 4) for the systems having RS values smaller than 2/16 the differences between isotherms vanish, however they appear for the systems with larger RS values, due to the filling of small pores $(\sim 0.3 \mathrm{~nm})$ between opposite bulges. A further rise in the distance between walls (series H3) causes the differences between adsorption isotherms to almost completely disappear. The rise in adsorption, in comparison to the reference system, is caused by the rise in adsorption induced by folding of walls. The appearance of pores $(\sim 0.6 \mathrm{~nm})$ between opposite bulges leads to differences in the plots of adsorption isotherms only in the intermediate range of relative pressures $\left(10^{-3}-10^{-2}\right.$, see also animation_04, 05 and 06). With the rise in RS value the swing on adsorption isotherms in this range becomes more pronounced. The differences between isotherms at low pressures are confirmed by the values of Henry's (or Langmuir) constants which are collected in Table 2 and plotted in Figure S1 in online supplementary data. As above the rises in the values of $K_{H / L}$ reflect the regularities observed on Figure 4. The important influence of the narrowest pores appearance and/or high-energetic places between neighbouring fragments of walls [I don't understand this - rewrite] on the slope of initial parts of isotherms is also confirmed by the values of isosteric enthalpy of adsorption presented en in Figure 5. The differences in $q^{\text {st }}$ values for low adsorption amounts fully correspond to the changes in the shapes of isotherms. 


\subsection{Adsorption of mixtures}

As shown above carbons with folded walls have better adsorption properties towards $\mathrm{Ar}$ than ideal slit-like pores. The same can be stated about separation properties. From Figures 6-8 collecting the values of equilibrium separation factors for the considered mixtures at the total pressure equal to $0.1 \mathrm{MPa}$ and different compositions one can see that generally with the rise in $H_{\text {eff }}$ the separation factors decrease. The increase in interlayer distance is also connected with reducing (H2 series) or vanishing ( $\mathrm{H} 3$ series) differences between the systems with different mutual orientation of the walls. The geometry of systems for $\mathrm{RS}=0 / 16$ is analogous to that in our previous paper [...]. In the case of the systems for $A=0.50 \mathrm{~nm}$ the interlayer distances are similar in the current and in the previous work. So, the similar values of equilibrium separation factors are observed. For the other values of $A$ (i.e. 0.75 and $1.00 \mathrm{~nm}$ ) the higher interlayer distances were necessary to allow unrestricted shift of the walls. Thus, the lower values of equilibrium separation factors are observed in this work.

The analysis of separation factors for the H1 series (Figures 6-8) leads to the conclusion about similarities in the plots of separation factors for $\mathrm{CO}_{2} / \mathrm{N}_{2}$ and $\mathrm{CO}_{2} / \mathrm{CH}_{4}$ mixtures. Similarly to the case of Ar adsorption, the best separation properties are recorded for the systems having $\mathrm{RS}=2 / 16$, and just like in the case of Ar adsorption, this is caused by the appearance of small pores preferentially adsorbing $\mathrm{CO}_{2}$ molecules. The systems having larger $\mathrm{RS}$ values $(\mathrm{RS}=3 / 16$ and 4/16) show worse separation properties than for $\mathrm{RS}=2 / 16$. In the case of $\mathrm{H} 1$ series for $\mathrm{A}=$ $1.00 \mathrm{~nm}$ the differences between separation factor values are not large. Here a similar situation as in the case of Ar adsorption is observed, i.e. adsorption properties are determined mainly by the internal parts of curved surfaces (see the solid-fluid potential energy maps collected on Figure S2 and S5 in online supplementary data). A different situation is observed for the $\mathrm{H} 1$ series if the separation of a $\mathrm{CH}_{4} / \mathrm{N}_{2}$ mixture is considered (Fig. 8). In this case, a significant rise in separation efficiency is observed starting from RS $=1 / 16$. The further rise in RS values also increases the $S_{C H 4 / N 2}$ values and the maximum for $\mathrm{RS}=2 / 16$ is not observed for the case of this mixture. Differences observed in comparison to $\mathrm{CO}_{2} / \mathrm{N}_{2}$ and $\mathrm{CO}_{2} / \mathrm{CH}_{4}$ mixtures are caused by the larger diameter of preferentially adsorbed $\mathrm{CH}_{4}$ molecule. As a consequence even for small RS values the centres with higher methane adsorption energy are created (see Figs. S2, S5 and S8 in online supplementary data). The differences described above in mixtures adsorption mechanism at $p_{t o t}=$ 
0.1 MPa connected with mutual shift of walls for the $\mathrm{H} 1$ series are also illustrated on animation_07,08 and 0.9 in online supplementary data.

The increase in interlayer distance by $0.3 \mathrm{~nm}$ (series $\mathrm{H} 2$ ) leads to the disappearance of the differences between separation factor values (for all the studied mixtures) for $S$ values for RS up to $2 / 16$, and only the systems having larger values of RS (3/16 and 4/16) show better separation efficiency on the similar level as observed for the best H1 systems. As mentioned above, this is a consequence of the presence of small pores between opposite bulges.

Finally for the $\mathrm{H} 3$ series the RS value practically has no influence on separation efficiency. In this case the higher efficiency in comparison to the ideal slit-like systems is determined by the adsorption on surface (higher adsorption energy on folded sheets).

Figure S11 in online supplementary data compares the values of equilibrium separation factors for equimolar gas mixtures adsorption $\left(p_{t o t}=0.1 \mathrm{MPa}\right)$ in all the considered systems. Figures 9-11 present isosteric enthalpy of all considered mixtures adsorption in all systems at 0.1 MPa (the data related to Figs 6-8). All curves are plotted as a function of the mole fraction of preferentially adsorbed component in the bulk phase $\left(y_{C O 2}\left(\mathrm{CO}_{2} / \mathrm{N}_{2}\right.\right.$ and $\left.\mathrm{CO}_{2} / \mathrm{CH}_{4}\right)$ ) or $y_{C H 4}$ $\left.\left(\mathrm{CH}_{4} / \mathrm{N}_{2}\right)\right)$. Since the energy of adsorption of this component is higher than of the second one, the rise in $y$ value causes the increase in $q^{s t}$ values. The differences in isosteric enthalpy of adsorption between different systems are analogous to those between the equilibrium separation factors shown in Figs 6-8. This confirms that the main reason respensible for the changes in separation efficiency connected with mutual shift of the walls, and/or increasing interlayer distance, is the appearance or disappearance of the narrowest pores between the neighbouring bulges. In such places the dominant component is adsorbed preferentially with high energy.

Finally, Figures 12-14 collect the equilibrium separation factors for the adsorption of equimolar mixtures (all considered) under different total pressure. The rise in the total pressure causes that adsorption takes to take place not only in highly-energetic places but molecules are also bonded on centres having lower adsorption energy. Multilayer adsorption (or even total filling of accessible pores) is also possible especially in the case of $\mathrm{CO}_{2}$ since the considered temperature $(298 \mathrm{~K})$ is below the critical value for this gas $(304 \mathrm{~K})$. Thus, the rise in total pressure value is usually connected with some increase in the equilibrium separation factors. This is caused by the enhanced disproportion between adsorption of dominant and less adsorbed components. There are some exceptions related to the adsorption of $\mathrm{CO}_{2} / \mathrm{N}_{2}$ and $\mathrm{CO}_{2} / \mathrm{CH}_{4}$ 
mixtures in the A0.50H1.08RS2/16 system. In these cases maxima on the curves in low pressure region are observed. Similar (but less pronounced) behaviour is also observed for A0.75H1.52RS2/16 and A0.50H1.38RS4/16. As it-was discussed above, high selectivity of mixtures adsorption in these structures is connected with the appearance of narrow pores between opposite bulges. The rise in the total pressure eatses means that adsorption takes place not only in these optimal places but also in the remaining pores where selectivity is not as high. In consequence, average value of equilibrium separation factor decreases. Next it slightly increases for $p_{t o t}$ close to $1 \mathrm{MPa}$ due to multilayer adsorption of $\mathrm{CO}_{2}$. Comparing the effects of mutual shifting of walls and/or of increase in the interlayer distance for all the systems (Figs 12-14) one can see analogieal analogous regularities as described above for adsorption of mixtures at $p_{\text {tot }}=$ 0.1 MPa (Figs 6-8). However, some differences are observed for adsorption of $\mathrm{CO}_{2} / \mathrm{N}_{2}$ and $\mathrm{CO}_{2} / \mathrm{CH}_{4}$ mixtures in $\mathrm{A} 0.50 \mathrm{H} 1.08 \mathrm{RS} 0 / 16, \mathrm{~A} 0.50 \mathrm{H} 1.08 \mathrm{RS} 1 / 16$ and $\mathrm{A} 0.75 \mathrm{H} 1.52 \mathrm{RS} 1 / 16$ systems for the total pressure close to $1 \mathrm{MPa}$. In this region the values of equilibrium separation factors are similar to these observed for systems with $\mathrm{RS}=4 / 16$. This behaviour may be explained by comparison of the pore sizes of these structures (Figs 2 and 3). In the case of the systems with small RS value dominant pores have smallest diameter (ca $1 \mathrm{~nm}$ for A0.50H1.08RS0/16 and A0.50H1.08RS1/16, ca $1.5 \mathrm{~nm}$ for A0.75H1.52RS1/16) than these in systems with high RS (ca 2 $\mathrm{nm}$ for $\mathrm{A} 0.50 \mathrm{H} 1.08 \mathrm{RS} 4 / 16$ and ca $2.3 \mathrm{~nm}$ for $\mathrm{A} 0.75 \mathrm{H} 1.52 \mathrm{RS} 4 / 16)$. In consequence, $\mathrm{CO}_{2}$ may fully fill pores of the first structures while it is not possible in the case of the second ones. Aniamtion_10, 11 and 12 in online supplementary data present the mechanism of $\mathbf{r}$ equimolar mixtures adsorption at different total pressure values for all the H1 systems.

Summing up, a mutual shift of the folded graphene sheets forming slit-like pores is connected with the formation of small pores. This fact (due to overlapping of interactions with opposite walls) causes a significant increase in adsorption energy and leads to a rise in the efficiency of mixture separation in comparison to ideal slit-like systems. The rise in interlayer distance is connected with the reduction or disappearance of differences between systems with different shifts. This is caused by the vanishing of effects connected with overlapping of interactions with the walls which is demonstrated on solid-fluid energy maps for $\mathrm{CO}_{2}, \mathrm{CH}_{4}$ and $\mathrm{N}_{2}$ molecules presented in Figures S2-S10 in online supplementary data.

\subsection{Comparison with other systems}


To show a comparison with other systems, we chose the mixture $\mathrm{CO}_{2} / \mathrm{CH}_{4}$ Figure 15 presents a comparison of equilibrium separation factors for the system having the most promising separation properties (A0.50H1.08RS2/16) and for adsorption inside ten single-walled carbon nanotubes $((15,0),(16,0),(17,0),(18,0),(19,0),(20,0),(21,0),(22,0),(23,0)$ and $(24,0))$ having diameters in the range $0.826-1.526 \mathrm{~nm}$ (the simulated results are taken from our previous study $[\ldots])$. As one can see our structure has remarkably larger $S_{C O 2 / C H 4}$ values than a $(24,0)$ nanotube with similar pore diameter as the dominant pores in our structure (c.a. $1.5 \mathrm{~nm}$ - see Fig. 2). Due to the formation of narrow pores between opposite bulges separation efficiency is comparable with this that observed for nanotubes having much smaller diameters. Bifferent A different situation is observed if we compare the results with those calculated for three virtual porous carbon (VPC, denoted as $\mathrm{d} 0.5, \mathrm{~d} 0.9, \mathrm{~d} 1.3$ - for their characteristics see [...]) models having remarkably very different microporosity (the simulated results for VPCs are taken from our previous study [..]) shown on Figure 16 . The d0.5 carbon has similar diameters of micropores as the A0.50H1.08RS2/16 structure, and two remaining carbons have smaller micropore diameters. It is seen that A0.50H1.08RS2/16 shows better separation properties than the d0.5 structure and similar to this that observed for the d0.9 one. Due to very smaller micropores present in $\mathrm{d} 1.3$ the separation properties of this structure are the best.

Summing up, our results show that the structures studied in this work containing pores with folded graphene walls should reveal better separation properties than single-walled carbon nanotubes and activated carbon having similar pore diameters.

\section{Conclusions}

We present the results of systematic Monte Carlo studies on the influence of mutual shift of walls in slit-like systems constructed from folded graphene sheets on the mechanism of argon 
adsorption (at its boiling point) and on the adsorption and separation of binary gas mixtures (at room temperature). We demonstrate that folding of the walls significantly improves the adsorption and separation properties in comparison to ideal slit-like systems. Moreover, we show that mutual shift of sheets (for small interlayer distances) causes the appearance of small pores between opposite bulges which causes an increase in Ar vapour adsorption at low pressure. Due to the overlapping of interactions with opposite walls causing an increase in adsorption energy the mutual shift of sheets is also connected with the rise in efficiency of mixture separations. The effects connected with sheet orientation vanish as the interlayer distance increases.

The presented results show that the structures studied in this work containing pores with folded graphene walls are very promising since they may reveat display better separation properties than single-walled carbon nanotubes and activated carbon having similar pore diameters.

\section{Acknowledgments}

SF, APT and PAG acknowledge the use of the computer cluster at Poznań Supercomputing and Networking Centre (Poznań, Poland) as well as the Information and Communication Technology Centre of the Nicolaus Copernicus University (Toruń, Poland). SF gratefully acknowledges financial support from Iuventus Plus Grant No. IP2012 034872 from the Polish Ministry of Science and Higher Education.

\section{References}

[1] Lu A-H and Dai S (ed) 2014 Porous Materials for Carbon Dioxide Capture (Berlin, Heidelberg: Springer-Verlag)

[2] Inagaki M and Kang F 2014 Materials Science and Engineering of Carbon: Fundamentals (Amsterdam: Elsevier)

[3] Furmaniak S, Terzyk A P, Gauden P A, Kowalczyk P and Harris P J F 2014 J. Phys.: Condens. Matter 26485006

[4] Liang C and Dai S J 2006 J. Am. Chem. Soc. 1285316

[5] Bhattacharya S and Gubbins K E 2006 Langmuir 227726

[6] Furmaniak S 2013 Comput. Methods Sci. Technol. 1947 
[7] Furmaniak S, Terzyk A P, Gauden P A, Kowalczyk P, Harris P J F and Koter S $2013 \mathrm{~J}$. Phys.: Condens. Matter 25015004

[8] Yan Q and de Pablo J J 1999 J. Chem. Phys. 1119509

[9] Do D D and Do H D 2005 Fluid Phase Equilib. 236169

[10] Nguyen T X 2006 Characterization of Nanoporous Carbons PhD Thesis University of Queensland, Brisbane

[11] Terzyk A P, Furmaniak S, Gauden P A and Kowalczyk P 2009 Adsorption Sci. Technol. 27281

[12] Potoff J J and Siepmann J I 2001 AIChE J. 471676

[13] Steele W A 1974 The Interaction of Gases with Solid Surfaces (Oxford: Pergamon)

[14] Frenkel D and Smit B 1996 Understanding Molecular Simulation from Algorithms to Applications (London: Academic)

[15] Tylianakis E and Froudakis G E 2009 J. Comput. Theor. Nanosci. 6335

[16] Furmaniak S, Kowalczyk P, Terzyk A P, Gauden P A and Harris P J F 2013 J. Colloid Interface Sci. 397144

[17] Furmaniak S, Koter S, Terzyk A P, Gauden P A, Kowalczyk P and Rychlicki G 2015 Phys. Chem. Chem. Phys. 177232

[18] Furmaniak S, Terzyk A P, Gauden P A, Harris P J F and Kowalczyk P 2009 J. Phys.: Condens. Matter 21315005 